\title{
Miguel de Unamuno sul fronte italiano: mito e propaganda della Grande Guerra, tra aristocrazia intellettuale e ceti popolari
}

\author{
Gabriele Morelli \\ Università di Bergamo
}

Fecha recepción 20.10.2014 / Fecha aceptación 06.04.2015

\section{Abstract}

L'articolo affronta ed illustra il tema dell' interventismo dell' Italia nella Grande Guerra, sostenuto dalle elites culturali, politiche e militari contro l' interesse e la volontà delle masse popolari e rurali del Paese, per lo più estranee al mito eroico della guerra. In particolare lo studio presenta il diario del viaggio di Miguel de Unamuno, invitato nel 1917 dal governo italiano a visitare il fronte militare; diario composto di vari articoli apparsi in gran parte sul giornale La Nación di Buenos Aires, insieme ad altri interventi pubblicati su periodici spagnoli e italiani. La figura di Unamuno, fervente sostenitore dell' interventismo contro le forze belligeranti della coalizione tedesca, è accolta con simpatia dagli intellettuali italiani, lettori entusiasti del suo commento al Don Chisciotte, visto quale interprete moderno dell' idealismo. Propaganda, misticismo eroico e visione letteraria finiscono per sovrapporsi nelle pagine del reporter Unamuno sul fronte italiano, mitigando la dolorosa immagine segnata dalla Grande Guerra con le sue contraddizioni e violenze.

\section{Parole chiave}

Unamuno, diario, interventismo dell'Italia, Grande Guerra, elites, masse popolari.

\section{Abstract}

This article focuses on the theme of the Italian intervention in World War I, which was supported by the cultural, political and military elite against the interests and the will of the popular and rural masses, mostly unrelated to the heroic myth of the war. In particular, the study presents the travel diary of Miguel de Unamuno, who in 1917 was invited by the Italian Government to visit the military front. The diary includes various articles, most of which appeared in the newspaper La Nación of Buenos Aires, and other works published in Spanish and Italian periodicals. Unamuno was portrayed as a fervent supporter of interventionism against the belligerent forces of the German coalition, and he was greeted with sympathy by Italian intellectuals, who were enthusiastic readers of his commentary on Don Quixote, seeing him thus as a modern interpreter of idealism. In the pages of Unamuno, there is overlapping propaganda, heroic mysticism and literary vision, mitigating the painful images of World War I with its contradictions and violence.

\section{Key words}

Unamuno, diary, Italian Intervention, Great War, elites, popular masses 
Nell' ambito del tema generale «Elites y pueblo», in cui si inseriscono queste giornate di studio del simposio dedicato a La Guerra di Carta: Letteratura e testimonianze sulla crisi della Prima Guerra Mondiale, non c’è dubbio che lo scrittore Miguel de Unamuno svolge un ruolo fondamentale a favore della partecipazione militare dell' Italia nella guerra contro la Germania. Occorre inoltre dire che la relazione di stima ed ammirazione del grande filosofo spagnolo nei confronti del nostro Paese e, in particolare, della sua cultura umanistica è antica e risale al suo viaggio giovanile compiuto nel 1889, subito dopo aver terminato gli studi universitari, attratto dal fascino della storia, la letteratura e l'arte italiana. Successivamente il legame tra Unamuno e gli intellettuali italiani si intensifica ed arricchisce in seguito alla pubblicazione delle sue opere, tra cui La vida de Don Quijote y Sancho, tradotto e diffuso in Italia, insieme ai suoi libri più importanti, che subito ricevono una grande accoglienza e un'immediata ricezione da parte dei giovani letterati ma anche di uomini illustri, come Benedetto Croce, Luigi Pirandello e Giovanni Papini ${ }^{1}$. Con il primo l'amicizia è testimoniata da un ricco rapporto epistolare che va dal 1911 al 1921, interrotto dall'esilio dello scrittore spagnolo durante il periodo della dittatura di Primo de Rivera, e che continua successivamente anche se in modo sporadico. Sappiamo inoltre che Don Miguel espresse più volte il desiderio di conoscere personalmente Croce, anche in occasione del viaggio in Italia per visitare il fronte italiano; come anche manifestò la volontà di incontrare e conoscere il grande scrittore toscano Giovanni Papini. In una sua lettera ad Ardengo Soffici, del 12 dicembre 1908, leggiamo: «Con Papini ho scambiato delle lettere ma non ho avuto il piacere di vederlo: e quanto mi gradirebbe vederlo costì... Quando vede Papini lo saluti caldamente a nome mio con una parola d' incoraggiamento» ${ }^{2}$. Allo stesso scrittore fiorentino, Don Miguel contesta la lettura che ha fatto del suo saggio sul Don Chisciotte, dove intende che Papini abbia tratto la convinzione della sua cattolicità, per cui scrive.

1. Relazioni e carteggi intercorsi tra Unamuno e i citati scrittori italiani sono stati ampiamente studiati da M. García Blanco, V. González Martín, G. Foresta e C. Luigi Ferraro. Su questi ed altri studi, si veda V. González Martín, La cultura italiana en Miguel de Unamuno, Salamanca, 1978. In particolare su Croce e Unamuno, si può consultare il recente libro di C. Luigi Ferraro, Benedetto Croce e Miguel de Unamuno, Città di Castello, 2004.

2. González Martín, op. cit., 227. 
«No, amigo Papini, no! no soy católico. Si fuera católico -como lo son en España, por lo menosni daría importancia a la religión ni me cuidaría del misticismo.

No sé qué pasajes de mi Vida de Don Quijote y Sancho han podido inducirlo al error de creerme católico. Allí, alguna vez me pronuncio por la fe de Sancho Panza, del pueblo rústico español, y pido a Dios que no le quite su fe en la inmortalidad personal, pero Sancho Panza, no es católico, no es católico de la iglesia del Papa» ${ }^{3}$.

Proprio la particolare lettura del saggio unamuniano sulla figura dell' Hidalgo crea in molti intellettuali italiani un fraintendimento che è possibile cogliere sul tema della Grande Guerra, alla cui causa lo scrittore spagnolo aderisce accogliendo l'invito del governo italiano a visitare il fronte. L'entusiastica adesione a favore della guerra, già caldeggiata dai maggiori rappresentanti della cultura italiana - si pensi a D’Annunzio, Marinetti e Papini - trova nel libro di Unamuno, interprete moderno del «chisciottismo», l' humus ideale che giustifica ed alimenta il sacrificio eroico a favore della patria. Lo stesso Papini, nel suo articolo dedicato all'amico spagnolo apparso su Il Leonardo, confessa l'intima adesione al legato spirituale in nome del Don Chisciotte:

«Io sento per lui una simpatia istintiva che è dovuta, probabilmente, alle somiglianze delle nostre anime e dei nostri scopi. Egli vuol fare per la Spagna ciò ch' io vorrei fare per l' Italia e riconosco pure come mio principal patrono l'immortale Don Chisciotte» ${ }^{4}$.

E non lesina la sua grande ammirazione al maestro spagnolo, a cui sente di essere vicino e di cui condivide pienamente il suo idealismo nel nome di Don Chisciotte:

«Miguel de Unamuno è oggi il sacerdote principe della Religione di Don Quijote di cui sono, per mia gloria e fortuna, un fervoroso fedele e ho provato il bisogno, appena l' ho scoperto, di mandargli il saluto del suo fratello ignoto, in questa rivista che si onora d'esser l'organo del don Chisciottimo italiano» ${ }^{5}$.

Al pari di Papini numerosi altri intellettuali, al momento della decisione ma anche dopo la partecipazione all'evento bellico, vedono nel libro di Unamuno un punto di riferimento spirituale a conforto della loro scelta interventista. Tra le numerose testimonianze si possono indicare molte lettere di giovani italiani dirette allo scrittore spagnolo che confessano l'influenza salutare esercitata dalla lettura del suo saggio sull'opera cervantina. È il caso, tra i tanti, del sottotenente Tommaso Fiore, impegnato sul fronte militare il quale, nell' inviare al maestro il suo articolo «L'esortazione», confessa che si tratta di una «manifestazione di donchisciottismo». Nella sua missiva del 21 ottobre 1917, così commenta: «Ho avuto buon

3. González Martín, op. cit., 225.

4. Il Leonardo, Firenze, ottobre-dicembre, 1906, 364-366. Citato anche da González Martín, op. cit., 224.

5. González Martín, op. cit., 225. 
compagno giù per i fossati di Doberdò e poi sullo sperone del Faiti il suo Commento al Don Chisciotte: questa offerta mia Le sia di ringraziamento» ${ }^{6}$. Come si vede, il libro che sostiene il giovane sottotenente, aiutandolo a superare le sofferenze e atrocità della guerra, è l'opera di Don Miguel. Lo stesso Tommaso Fiore in una missiva successiva, datata 26 settembre 1918, inviata dalla prigionia tedesca di Gefangenenlanger Schwarmstedt in provincia di Hannover, torna a scrivere a Unamuno:

«Illustre Signore,

A un anno di distanza mi ritrovo prigioniero! Ho qui qualche buon compagno, tra cui il suo commento al Don Chisciotte, e veramente mi vado chisciottizzando sempre più. Di questo spero che darà subito qualche frutto, anche migliore, se non come arte, come pensiero, di quelli antecedenti. Ha ricevuto l'anno scorso la mia Esortazione? O è rimasta per istrada? Avrei caro saperlo, perché vorrei da Lei esser armato buon cavaliere, onesto e prode, e di qualsiasi figura. Si conservi sano pel bene del suo paese e nostro e se può e non l'è di noia mi scriva qualche volta. E quando potrò conoscerLa personalmente? Ma ora fo' penitenza come il buon Hidalgo. Coi sensi della maggiore considerazione» ${ }^{7}$.

Ricorrente è la menzione al commento unamuniano dell'opera maestra di Cervantes che incarna l'eroismo utopico e solitario contro la violenza e la barbarie, rappresentate queste dal fronte espansionista della Germania. Soprattutto incessante è il richiamo alla lettura del libro e alla figura dello scrittore in quanto interpreti superiori di una missione idealistica che, nel frangente dello scontro militare, si tinge di valori spirituali e religiosi. Un esempio eloquente viene da una nuova testimonianza epistolare inviata il 6 febbraio 1917 al maestro salmantino dal giovane tenente Gino Argan, poco dopo il viaggio compiuto da Don Miguel sul fronte italiano, di cui trascrivo i passi più significativi che documentano l'abbandono e la completa fiducia nei confronti di Unamuno:

«Vi sono necessità degli spiriti che non si discutono. Spesso cerchiamo una persona con cui confidarci, per parlare a noi stessi.

Ho letto il vostro Commento al Don Chisciotte, subito dopo la storia della sua mirabile vita scritta da Don Miguel Cervantes e contemporaneamente al vangelo di N. S. Gesù Cristo. Voi dite che sareste felice se il vostro libro avesse messo la battaglia e l'affanno in qualche cuore addormentato, come quasi tutti, nel torpido equilibrio e nella quiete oziosa delle dottrine sillogistiche.

Qualche cosa di simile ha ottenuto in me il Cavaliere Don Chisciotte per mezzo vostro. Non ha suscitato affanni ma li ha risvegliati, non ha creato regni, ma li ha rivelati al mio spirito, regni di bellezza e di luce. Io sono di fatto intimamente chisciottista, più di quel che non lo si sia ai miei 20 anni, e il mio chisciottismo attinge alla più angusta fonte di ogni nobile attitudine: a mia Madre.

6. G. Foresta, “Unamuno interventista”, Nuova Antologia, 2073, settembre, 1973, 72.

7. Foresta, op.cit., 72. 
[...] In questa Roma, ove attendo impaziente di andare al fronte, mi sento vicino spiritualmente al vostro, al nostro Cavaliere Don Chisciotte. Ho detto nostro, perchè se me lo permettete, io credo di ravvisare il lui il superbo temperamento latino $[\ldots] \aleph^{8}$.

Il giovane tenente, animato da grande ardore patriottico e con la mente rivolta all'eroe rappresentato dalla figura del Don Chisciotte, non esita a muovere una dura critica contro coloro che si imboscano negli uffici, sfuggendo in modo ipocrita e truffaldino al richiamo delle armi, mentre ammira e elogia gli umili rappresentanti del ceto popolare - i nostri Sancio Pancia, li chiama - che «vanno sereni alla lotta e combattono disperatamente, convertiti interamente alla fede della Patria». Dovrebbe vedere, indica ancora a Unamuno, come questi nostri contadini vanno incontro alla morte «per pura fede senza comprendere di storia e di politica, senza sperare dalla guerra alcun beneficio, allora Vi convincereste che Don Chisciotte è anche un po' nostro, latino!»?.

Il giovane ufficiale è spinto e motivato nei suoi giudizi, come confessa, da un intimo e nobile bisogno spirituale. Ma è proprio la dichiarazione di tale convincimento che lascia perplessi soprattutto allorché egli crede di interpretare anche la volontà dei ceti popolari i quali, sebbene sottoposti dal potere ufficiale (compreso quello religioso) a una ossessiva e prevaricante campagna propagandistica, restano per lo più estranei al mito eroico della patria, date le condizioni di miseria e analfabetismo in cui versano e sono costretti a vivere. Dobbiamo ricordare che le masse rurali del Mezzogiorno e anche quelle del sottoproletariato urbano, strappate dalle campagne e dalle loro povere case e famiglie, sono costrette a combattere per un ideale che è lontano dalle loro preoccupazioni reali, e che in ogni modo non possono comprendere e condividere. In realtà di questi «Sancho Panza», umili e generosi eroi della guerra, come li vede a modo suo Gino Argan nella sua missiva ad Unamuno, la letteratura non si è molto interessata o ha lasciato molte testimonianze, al contrario per lo più sono stati ignorati dell'elite culturale e militare interessata a restituire un'immagine edificante della partecipazione popolare alla guerra: «Patria o morte!», come recitano numerosi proclami dell'epoca. Del resto, è ben noto, le autorità civili, militari e religiose fecero ricorso a un impiego massiccio e sistematico della propaganda e della censura per giustificare o nascondere all'opinione pubblica le terribili scelte e conseguenze dovute all'evento bellico. Esistono infatti pochi documenti di denuncia, oltre a quello rappresentato dell' incerto carteggio dei poveri fanti sfuggito all'occhio rapace della censura militare, che testimoniano la terribile situazione sofferta dai militari come dai civili, e che documentano il malessere e il rifiuto del fante italiano nei confronti della terribile guerra di trincea. Tra questi si distingue il film, capolavoro del cinema italiano, La Grande Guerra del 1959, diretto da Mario Monicelli e interpretato da Alberto Sordi e Vittorio Gassman, due antieroi umanissimi (il primo, Sordi, eroe vigliacco, e il secondo, Gassman, eroe spavaldo), che mostrano insieme alla terribile assurdità della guerra, le condizioni di vita miserevoli dei militari e della gente. Soprattutto il film reagisce alla propaganda divulgata prima e dopo il fascismo e nel secondo dopoguerra, basata sul mito

8. Foresta, op.cit., 73.

9. Foresta, op.cit., 73. 
popolare di una Italia eroica; film che infatti creò problemi con la censura e fu vietato ai minori di 18 anni. Al contrario La Grande Guerra presentava, tra scene comiche e drammatiche, tipiche della commedia italiana, la vera immagine della terribile vita di trincea in cui commilitoni e semplici ufficiali appaiono in forte contrasto con i comandi superiori e le istituzioni ufficiali, indicate come le principali responsabili di quel massacro. Analoga denuncia contro la follia e la violenza della Grande Guerra è espressa dal film Uomini contro del 1970, diretto da Francesco Rosi, liberamente ispirato al romanzo Un anno sull'Altipiano di Emilio Lussu. La pellicola fu denunciata per vilipendio dell'esercito e a lungo boicottata, a dimostrazione dell' immagine consacrata dal potere, considerata frutto della partecipazione popolare.

\section{Solidarietà di Unamuno verso l'Italia e interventismo contro il germanesimo belligerante}

Torniamo in Italia al momento dell'evento bellico e dell' intervento a fianco degli alleati contro i germanofili, dove fra i primi si schiera Miguel de Unamuno, mentre la Spagna dichiara la sua neutralità ma vede nascere al suo interno da una parte un partito di intellettuali sostenitori dell'Alleanza latina, fra i quali oltre a Don Miguel, si schierano gli scrittori Pérez Galdos, Azorín e Valle Inclán; dall'altra parte troviamo Pio Baroja e Gustavo Benavente che parteggiano per i tedeschi. A tale divisione, e in particolare all'ingiustificata e forse interessata neutralità della Spagna, già alludeva Giovanni Papini nel suo articolo «Cosa fa la Spagna?» - titolo poi ripreso dallo stesso Unamuno -, apparso su Il Resto del Carlino (Bologna, 27 maggio 1915), in cui invita il paese iberico a aderire alla guerra accanto all' Italia e agli Alleati, denunciando al contempo le promesse fatte dalla Germania alla Spagna che assicuravano, in compenso della sua neutralità, la roccaforte di Gibilterra, il Marocco e persino il Portogallo. Nel citato articolo di Papini non manca l'elogio all' interventismo di Unamuno a favore delle nazioni latine «contro la Kultur», come scrive l'autore, che ricorda: «In nessun paese, forse, sono state dette così forti e atroci parole contro il tedescun come quelle di Miguel de Unamuno, il pensatore che anche in Italia, per merito mio, si comincia a conoscere e a leggere». L'eco dell'attività propagandistica svolta dagli intellettuali spagnoli e in particolare quella intrapresa con forza e convinzione dallo scrittore filosofo a favore del nostro paese è accolta con soddisfazione da alcuni ispanisti italiani, come Lucio Ambruzzi che nel luglio del 1915, così scrive a Unamuno:

Ilustre amigo,

Acabo de leer en un diario que los literatos españoles han lanzado un manifiesto para afirmar su solidariedad con la civilización contra la barbarie y el salvajismo disfrazados de Kultur (con inicial cuadrúpeda, como Ud. felizmente la bautizó). Esta noticia me llena de gozo, neutralizando la pesadumbre que me había causado la otra de que algunos diarios españoles (españoles de 
idioma pero no de carácter) se encarnizaban contra Italia por su intervención en la guerra y no al lado de sus fedífragos ex aliados ${ }^{10}$.

Nel riferimento fatto dall' ispanista ad alcuni giornali spagnoli sostenitori della causa tedesca, Ambruzzi allude chiaramente all' $A B C$ ed El correo español di Madrid, organi di stampa della destra cattolica vessilliferi del gruppo dei germanofili. Intanto la notizia dell' intensa attività propagandistica di Unamuno - oltre alla sua partecipazione a numerosi comizi pubblici tenutosi a Madrid nel 1917 in opposizione alla neutralità della Spagna, ed aspramente criticati dall' $A B C$ - è in più occasioni ripresa e diffusa dalla stampa spagnola come anche da quella italiana. Il nuovo giornale di Firenze (29 maggio) così titola: «Gli spagnoli contro l'imperialismo tedesco - Importante comizio spagnolo a favore dell' Italia - Si reclama la guerra alla Germania»; mentre, nello stesso giorno, La Tribuna di Roma scrive: «Un comizio a Madrid in favore dell' Intesa - Vi partecipa Unamuno». Altri interventi di Unamuno a favore dell'Intesa, a compendio ed integrazione degli articoli sul suo viaggio sul fronte italiano, sono i tre prologhi alla versione spagnola delle opere Historia ilustrada de la guerra de 1914 di Gabriel Hanotaux; Yo acuso di uno autore anonimo tedesco, e La ciudad doliente - Diario de un soldado raso di Gastón Riou. A questo proposito osserva Gaetano Foresta: «Unamuno non risparmia i suoi attacchi all'imposizione dogmatica e tecnicista della Kultur tedesca» che, secondo lo scrittore spagnolo, «pretende di dominare la libera coscienza dei popoli solo con la forza delle armi» ${ }^{11}$, e critica con ironia e vivace asprezza i connazionali difensori della causa tedesca, come nel seguente passo:

«Per il popolo tedesco il popolo spagnolo non esiste se non tutt'al più come un popolo incivile $o$ addomesticato, di sangue caldo che vive suonando la chitarra e prendendo il sole tra aranceti e limoneti. La germanofilia serve soltanto di pretesto per la diffusione delle peggiori passioni nazionali, dei più bassi istinti della nostra casta, del culto inumano della violenza autoritaria, dell'odio alla libera ed eretica personalità umana. La germanofilia è la meccanizzazione e metodizzazione della vita; il formale che sommerge ciò che è fondamentale, la morte spirituale della libera personalità umana, la società civile trasformata in un formicaio o alveare» ${ }^{12}$.

Ancora, nel prologo citato all'edizione spagnola del libro Yo acuso, possiamo leggere:

«I nipoti barbari che ci descrisse Tacito nelle sua Germania andavano sostituendo Wotan e Thor con Cristo e Platone, ed ecco che improvvisamente ritornano alla loro selva nera, ma a una selva irta di cannoni. Hanno appreso la scienza, ma hanno dimenticato la morale senza la quale la scienza non è se non barbarie raffinata. Perché la morale, nonostante l'imperativo categorico, non è matematica. La morale è simpatia. E la cosa più profonda della simpatia umana è non pretendere di dominare gli altri. Il tiranno, sia uomo o popolo, si nutre delle carni dello

10. Foresta, op. cit., 83.

11. Foresta, op.cit., 79. Per la lettura dei tre prologhi si veda M. de Unamuno, Obras completas, VIII, Madrid 1958, 1050-1061, 1062-1066, 1078-1083.

12. Foresta, op. cit., 79. 
schiavo. Così si spiega che un popolo servile pretenda di ridurre gli altri popoli a servitù. Vuole uguagliarli tutti nella sua abiezione. Perché devono servire gli altri, liberi, quando esso non lo è?

[...] Io capisco questo popolo servile e sottomesso, condotto al macello da una casta di potenti della spada, con il Kaiser alla testa; povero popolo che avanza fianco a fianco per essere mitragliato... che non ha coscienza né coraggio di liberarsi» ${ }^{13}$.

Il giudizio severo di Unamuno sul comportamento contraddittorio dell'elite intellettuale spagnola è anche condiviso dalla stampa francese, in particolare da Raymond Lautier, nei due interventi apparsi il 16 luglio 1916 e 1 luglio 1918 sul Mercure de France ${ }^{14}$, che criticano l'interessata estraneità del paese iberico, il quale, nei settori della società reazionaria, mostra una chiara simpatia nei confronti della politica aggressiva della Germania. In ogni modo, appassionata e radicale continua la lotta di Unamuno contro la volontà militaresca dell' impero germanico e ciò allo scopo di scuotere le coscienze culturali e politiche del suo Paese, allontanandosi in questo dalla posizione di Benedetto Croce. In effetti il grande filosofo italiano, in particolare nelle sue note «Malumori antihegeliani»e "Germanofilia» ${ }^{15}$, difende apertamente la cultura tedesca, motivato anche dalla manifesta ammirazione per la filosofia e la letteratura tedesca, di cui a lungo si è nutrito. La differenza del pensiero fra i due grandi scrittori è notevole ed appare con evidenza in un articolo di Unamuno intitolato Mameli e Körner, uscito su Il nuovo giornale, l' 11 gennaio 1916, dove di fronte all'esaltazione del poeta tedesco del misticismo guerresco germanico basato sul numero e la forza, Unamuno, prendendo spunto dallo studio di Carducci dedicato a Goffredo Mameli, oppone al modello tedesco la fede e l'eroismo del popolo italiano cantato dal poeta e patriota nazionale. Don Miguel torna sull'argomento nell'articolo "A proposito di alcune lettere di Chesterton a un garibaldino», sempre pubblicato su Il nuovo giornale, il 17 marzo 1916, in cui mette a confronto la filosofia pagana della dottrina dell'impero tedesco di fronte all'azione solitaria e generosa dell'eroe risorgimentale italiano, Giuseppe Garibaldi. Scrive Unamuno:

«Essi sono il popolo scelto da Dio, dal vecchio dio tedesco, da un dio delle battaglie, da un Elshim Sabaoth di un Jejovah sinaitico. Nel fondo è paganesimo puro; poiché tutte quelle atrocità di popoli superiori e inferiori, di razze decadenti, di nazioni chiamate a comandare le altre e di altre chiamate a essere comandate, non sono che paganesimo e mancanza di senso della libertà » ${ }^{16}$.

Dalla polemica in corso si evince che il dibattito è relegato all' interno del circolo aristocratico della militanza intellettuale e del potere politico-economico, cioè esso appare lontano dal coinvolgimento e dall' interesse avvertito dalle masse popolari, come sempre distanti ed

13. M. de Unamuno, "Prólogo a II ed., Yo acuso por un alemán, op. cit, 1065.

14. Rispettivamente: M. de Unamuno, "L'attitude des intellectuels espagnols dans le conflit européen. L'information et la litterature de guerre", Mercure de France, 16 luglio, 1916; e M. de Unamuno, "Quelques points de vue espagnols sur la guerre", Mercure de France, 1 luglio, 1918.

15. B. Croce, L'Italia dal 1914 al 1918. Pagine sulla guerra, Bari, 1950, 43, 68.

16. Vedi anche Foresta, op. cit., 82. 
estranee alla guerra, invocata invece dalla casta militare desiderosa di potere e, in particolare, caldeggiata dalle lobbies industriali avide di arricchimento in un momento in cui si assiste a una grande trasformazione tecnologica che riguarda la produzione di nuove armi e moderne macchine di guerra. Va anche detto che nell'ambito della discussione letteraria l'adesione all' intervento militare assume nel pensiero di Unamuno un carattere idealistico a difesa di valori assoluti affermati dalla cultura umanistica per i quali la guerra, secondo quanto afferma lo scrittore nel libro Del sentimento tragico della vita, trova una sua giustificazione necessaria per lottare contro il male e per il trionfo del bene superiore. Inoltre, alla frattura sorta tra la società pacifista latina e quella belligerante tedesca, Unamuno, sollecitato a dare la sua adesione alla nascita di organi di stampa, continua ad esaltare con immutata fede la difesa della nazione italiana. È sempre Gaetano Foresta ${ }^{17}$ ad informare di una lettera di Turati, datata Parigi 1927, inviata dall'esilio francese a Don Miguel a cui chiede «- ne plus que quelques lignes - d'adhésion, de salutation, de souhaites» al nuovo settimanale La libertà, fondato da Filippo Turati, Claudio Treves, Giovanni Amendola ed altri. Del resto Don Miguel, dall'amico traduttore Gilberto Beccari, aveva anche ricevuto nel 1913 la proposta di presiedere in Spagna l' "Associazione italiana della Lega Latina», poiché considerato il vero apostolo dell'anima spagnola, l'uomo ideale «más indicado a dar vuelo y poner en hechos idea tan grande y fraterna». La lettera inedita, datata 29 maggio 1913, conservata nell'archivio della casa museo Unamuno di Salamanca, propone:

«[...] reforzar, á medio propaganda, los vínculos de hermandad entre el pueblo italiano y aquellos de civilización latina, á fin de equilibrar los agrupamientos formidables que van formando en Europa pueblos eslavos y alemanes [...].

Educar el alma dividida frecuentemente de los pueblos latinos á unirse, á constituirse, á afirmar su genio vetusto á fin de renovar un núcleo de civilización que un día fué glorioso; para ofrecer á los demás pueblos de Europa mirable ejemplo de paz y de hermandad; prevenendo [sic] con la constitución del Mediterráneo en lago latino [...]. Es una obra de educación popular que se inicia, obra segura en un lejano porvenir contra las maniobras de las diplomacias18.»

Come si vede, si afferma la volontà di un proselitismo a favore dell'unità culturale dei paesi latini da parte di un gruppo di scrittori e politici: «Hombres resueltos y eminentes», precisa Gilberto Beccari, che si erigono a interpreti naturali dell'anima popolare, in realtà falsamente indotta o addirittura contraria all' idea della guerra, da sempre temuta poiché che li allontanava dalle preoccupazioni quotidiane e metteva in pericolo la loro stessa vita. Non meraviglia quindi che l'invito rivolto dal governo italiano a visitare il fronte militare venga accolto da Unamuno con grande favore. Con lo scrittore viaggiano altri insigni uomini della cultura spagnola: il grande studioso Americo Castro, il pittore Santiago Rusiñol, Manuel Azaña, futuro presidente della Repubblica spagnola,

17. Foresta, op. cit., 77.

18. Si riproduce alla fine il testo integrale della lettera. 
e Luis Bello, giornalista. Il racconto di Unamuno della sua visita ai campi di battaglia nella regione del Friuli darà luogo a sei articoli pubblicati su La Nación di Buenos Aires, materiale che insieme ad altri interventi a favore della guerra, oltre a quelli apparsi su $\mathrm{Il}$ nuovo giornale, escono sulle pagine dei giornali spagnoli La Publicidad di Barcellona e El Mercantil valenciano di Valenza. Articoli e discorsi pronunciati da Unamuno in occasione di presentazioni di alcune riviste - per esempio il settimanale «España»-, come pure i numerosi incontri a cui è chiamato a difesa della «Lega Antigermanica spagnola», si possono ora leggere anche in italiano nel recente libro Miguel Unamuno, L'agonia dell'Europa. Scritti della Grande Guerra ${ }^{19}$.

\section{II viaggio di Unamuno sul fronte italiano}

La visita di Unamuno inizia il 14 settembre del 1917: 28 anni dopo - ricorda lo scrittore - il suo primo viaggio italiano. Lo scrittore torna, confessa, in una nazione che mostra d'essere «anche maestra nell'arte della grande guerra moderna, della guerra industrializzata, come lo era stata nella strategia classica coi suoi condottieri e nel pieno della lotta per la causa dell'umanità civile, della civiltà umana, nella precoce e virile maturità nazionale $»^{20}$. L'interesse di Don Miguel per l'Italia è ora diverso: raccogliere le impressioni del nostro Paese in guerra e difendere l'intervento militare della nazione amica contro l'imperialismo espansionistico dell'esercito austro-ungarico e, soprattutto, spingere la Spagna ad uscire dalla sua posizione passiva per partecipare a fianco dell' Italia, erede della grande cultura latina ed interprete dell'autodeterminazione dei popoli. La difesa dell' interventismo italiano sembra inoltre rispondere all'appello lanciato nel 1916 da Giovanni Papini nel suo articolo citato «Cosa fa la Spagna?», cui fa eco l'analogo titolo di Unamuno che raccoglie la domanda che gli rivolgono con insistenza intellettuali e militari. Non meraviglia quindi che il suo arrivo in Italia sia salutato con grande entusiasmo dallo stuolo di scrittori amici ed estimatori, tra cui Mario Puccini, Ardengo Soffici, G.A. Borghese, Giovanni Amendola ed Ugo Ojetti, che intrecciano con il maestro spagnolo un ricco carteggio in gran parte inedito nella sua interezza, di cui offriamo due esempi significativi dell'attenzione con cui i giovani scrittori italiani interventisti accolgono l'autore spagnolo. Si tratta di due lettere: la prima dello scrittore Mario Puccini, soldato al fronte, che si duole di non aver potuto salutare degnamente lo scrittore; la seconda è del grande romanista Arturo Farinelli, che si cruccia di non essere stato avvertito dall'ammirato maestro del suo arrivo a Milano.

19. M. de Unamuno, L'agonia dell'Europa. Scritti della Grande Guerra, Milano, 2014. Cura e traduzione di Enrico Lodi, Da cui citiamo i passi utilizzati; si veda anche il saggio di Foresta, op. cit.

20. Lodi, op. cit., 23. 
Lettera di M. Puccini a M. de Unamuno ${ }^{21}$

[Carta intestata:] Comando della Brigata Veneto

[settembre-ottobre 1917]

Illustre Maestro ed Amico,

sono stato dolente di non potere, in occasione della Sua visita, mostrarle tutto il mio affetto, la mia ammirazione e quell'ospitalità ch' Ella tanto meritava.

Ma, come Ella capisce, io ero in mezzo ai miei Superiori: e, di più, io sono un timido e quasi un fanciullo.

Mi scriva, Maestro. Sarò tanto lieto di far conoscere agli Italiani la Sua opinione su questa nostra prova grandiosa - dalla quale l' Italia deve uscire ingigantita. Attendo le copie dei giornali pei quali Ella si è occupata delle mie Faville.

Ossequi e auguri vivissimi. Ora sono tornato in prima linea.

Suo dev.

Mario Puccini

Comando della Brigata Veneto

III Armata

Zona di guerra

Lettera di A. Farinelli a M. de Unamuno ${ }^{22}$

Belgirate (Lago Maggiore), 18 settembre 1917

Caro Signore,

Le scrissi una quindicina di giorni fa a Salamanca, ed ora leggo stupefatto nel Corriere della Sera che Ella passa da Milano (a due ore da Belgirate!) con alcuni colleghi ispanici diretti al nostro fronte!!!

Bravo! Senza degnarsi di comunicarmi la notizia del Suo viaggio! Che amicizia è trascurare me, piccolissimo ma forte studioso della Sua Spagna come nessuno dei miei illustri compatrioti?

21. González Martín, op. cit., 290.

22. G. Morelli, "Relazione letteraria. Farinelli-Unamuno", Cultura italiana e spagnola a confronto: anni 1918-1939, Tübingen, 1992. 
Io mi sarei subito mosso per incontrarla. Le avrei fatto gran festa!

Ora addio. A Salamanca prenderà questa mia e la getterà nel cestino.

Suo Arturo Farinelli

Occorre ancora ricordare la relazione epistolare dello scrittore e giornalista Ugo Ojetti, fondatore della rivista Pan, solerte diffusore e traduttore dell'opera unamuniana, al quale lo spagnolo invia dall'esilio di Fuerteventura una delle prime lettere di denuncia e rabbiosa protesta contro il dittatore Primo de Rivera. La missiva, spedita all'indirizzo del Corriere della Sera dove Ojetti lavora, non viene pubblicata: contiene violenti insulti contro il dittatore spagnolo («passa tutte le notti nei bordelli», «è un mostro di frivolezza, con un attacco di cretinismo tremens») e dunque potrebbe essere letta come una critica indiretta alla persona di Mussolini; da cui l'autocensura probabilmente imposta dallo stesso giornale milanese ${ }^{23}$.

Il reportage del viaggio si apre con l'arrivo di Unamuno a Milano, le visite rituali alla Certosa di Pavia, al Lago Maggiore e il racconto dell'ebbrezza del primo volo sulla città lombarda a bordo di un aereo della Caproni, i cui toni sembrano attingere alla retorica futurista. Ecco le impressioni di Unamuno che guarda dall'alto la città: impressioni vissute con spirito d'avventura dallo scrittore che sembra voler impartire una lezione sugli effetti positivi creati dal volo:

«Davvero è un piacere contemplare, seduto su di esso [l'aereo], uno di quei grandi incrociatori del cielo. E infonde un tale senso di sicurezza che mi sono deciso - alla mia età - a farmi portare in aria da un abilissimo aviatore italiano. Così ho potuto vedere Milano ai miei piedi, come una carta topografica, e il suo Duomo mille metri sotto di me, e la verde pianura lombarda che sembrava inclinarsi da una parte all'altra, quando in realtà eravamo noi a farlo.

La paura del volo? Molta meno di quanto ne avessi all' inizio. Il fatto è che l'animo si trova dapprima impacciato come da un senso di pericolo. Poi, quando uno si slancia in una dei quelle avventure in cui si pensa che sia concreta la possibilità di perdere la vita, la paura scompare e viene sostituita da un grande lucidità, come da una sollecita prontezza di tutti i sensi» ${ }^{24}$.

Le pagine successive conducono la delegazione spagnola al quartiere generale di Udine e all' incontro con il generale Luigi Cadorna, capo di stato maggiore dell'esercito italiano e principale fautore dell'intervento contro le truppe austro-ungariche. Nel gennaio del 1917, l'anno della visita italiana di Unamuno, Cadorna aveva preso parte alla «Conferenza Interalleata di Roma», cercando di convincere francesi e britannici ad inviare le loro divisioni in Italia, ma senza esito positivo. Il ritratto edulcorato che Unamuno traccia di Cadorna, conosciuto nel breve colloquio intercorso nell' incontro di Udine, sembra rispondere al canone

23. Si veda G. Morelli, "Una lettera inedita di Miguel de Unamuno: dal confino di Fuerteventura un vibrante appello alla stampa italiana”, Lingua e Letteratura, 4, Milano, 1985, 5-13.

24. Lodi, op. cit., 28. 
retorico imposto dalla propaganda ufficiale, sebbene è più facile credere che lo scrittore, nel clima di entusiasmo collettivo e infatuazione generale, sia portato sentimentalmente a condividerlo e a farlo proprio, cogliendo come sincero il rapporto di stima che lega il generale al popolo e viceversa. Leggiamo la pagina dedicata alla figura del capo supremo dell'esercito italiano, di cui Unamuno offre un ritratto fisico ma anche interiore:

«Cadorna ci è apparso come un uomo estremamente affabile, semplice e anche sereno. Più che sorridere, rideva di tanto in tanto, scoprendo una dentatura magnifica, di uomo sano e più giovane rispetto a quando corrisponde alla sua vera età. Quel volto energico e duro, ma anche limpido e aperto, volto da contadino, si accendeva di una luce interiore che era segno di fiducia in se stesso e nel suo popolo. Perché la maggiore risorsa del generale Cadorna pare essere la fiducia che nutre verso il suo popolo, così come la forza di questo popolo è la fiducia verso l'uomo che ha posto a capo del proprio esercito» ${ }^{25}$.

Il diario poi si sofferma a descrivere le acque trasparenti del fiume Isonzo e ancora le terre calcaree del Carso che a Unamuno ricordano l'arida meseta della vecchia Castiglia. Un racconto che guarda all' uomo e alla bellezza del paesaggio sconvolto dalle armi, e in cui, nonostante tutto, lo scrittore coglie un rapporto di serena, grandiosa maestà. Memorabili sono le descrizioni del sacro Isonzo con le sue «acque chiare e quasi cerulee», «le sue passerelle e i ponti della morte». L'evocazione del fiume richiama al lettore italiano i noti versi del poeta Giuseppe Ungaretti, allora soldato sul fronte, che ritrova nelle fresche acque dell' Isonzo la sua intima dimensione umana e spirituale in armonia con la natura:

«L' Isonzo scorrendo

Mi levigava

Come un suo sasso $[\ldots]$

Mi sono accoccolato

Vicino ai miei panni

Sudici di guerra

E come un beduino

Mi sono chinato a ricevere

Il sole

Questo è l' Isonzo

E qui meglio

Mi sono riconosciuto

Una docile fibra

Dell' universo».

25. Lodi, op. cit., 31-32. 
Più avanti, dalle alture del Monte Nero, Unamuno osserva il villaggio disabitato di Tolmino e, più in basso, la linea bianca del fiume che scorre lento, dove una natura selvaggia, ma al contempo mite, sembra lenire e cancellare gli orrori della tragedia umana:

«[...] tra burroni abissali, ai piedi di rientranze improvvise e di precipizi, serpeggiava il fiume Isonzo [...]. Lì la natura è selvaggia, ma lo è in modo sereno e persino dolce. Ritempra dagli orrori della bellicosa tragedia umana. Quella natura dà maggiore grandezza alla guerra, ma smussa anche la sua feroce brutalità. L'uomo lì ha lottato con l'uomo, ma lo ha fatto anche, in eguale misura o forse di più, con la natura stessa $»^{26}$.

Anche le cime del monte San Michele richiamano l'attenzione del viaggiatore spagnolo, mentre lo sguardo spazia in basso sulla città di Gorizia avvolta nella bruma del mattino. Il Vallone del Carso ricorda a Unamuno le aree minerali della sua Biscaglia, mentre intorno, nelle scarpate scavate nella pietra, osserva le baracche dei soldati intenti alle loro occupazioni sembrano alveari ronzanti. L'evocazione unamuniana del paesaggio lunare del San Michele, teatro di tante sanguinose operazioni militari che costarono migliaia di morti, ricorda al lettore italiano la nota poesia «Sono una creatura» di Ungaretti, in cui il grande poeta esprime il dolore per la guerra e per la morte di tanti compagni, rivendicando il suo essere umano, la presenza della ferita creata da tante morti e il prevalere del pianto, freddo e disanimato come la pietra che lo circonda:

\author{
«Come questa pietra \\ del S. Michele \\ così fredda \\ così dura \\ così prosciugata \\ così refrattaria \\ così totalmente \\ disanimata \\ Come questa pietra \\ è il mio pianto \\ che non si vede \\ La morte \\ si sconta \\ vivendo».
}

Il viaggio continua, tra soste e ritorni a Udine; lo scrittore si inerpica su ripidi camminamenti di guerra fino a raggiungere le alte trincee dello Zagradan, il monte Sabotino e quindi costeggiare in automobile le alpi dolomitiche. Seguono le visite a tre ospedali di guerra, in

26. Lodi, op. cit., 50 . 
particolare a quello stomatologico di Udine con l'immagine straziante dei volti feriti, i corpi mutilati dei soldati, relitti umani chiusi nel loro composto dolore. Un quadro del dolore - e dell'orrore - che a Unamuno, ispirato più dalla propaganda bellica che dalla pietas, appare «moderno, raccolto, metodico». Lo scrittore insiste sull' intervento militare inteso quale «agonia», lotta salvifica contro l'egemonia della Germania, ma la cui certezza, dopo le notizie della disfatta di Caporetto, pare incrinarsi gettando ombre sulla bontà del mito patriottico, strumento necessario di unità spirituale di una nazione.

Reporter di rango sul fronte italiano, Unamuno lascia un diario vivo, non di memoria, elogiato da Mario Puccini sulle pagine del Messaggero (16-11-1919) come «una delle più belle opere in prosa che la guerra abbia ispirato». Soprattutto il diario è il legato d'amore e di ammirazione che il grande scrittore spagnolo sente per l' Italia, i suoi uomini e la sua cultura. 


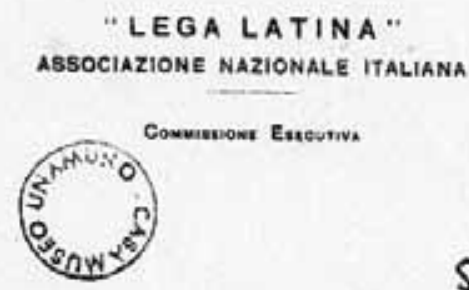

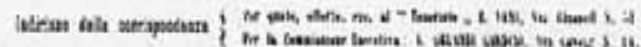

$$
\begin{aligned}
& \text { Hiz-2 Eisnas, E } 29 \text { demayor. } 1915
\end{aligned}
$$

Sr. D. Higuel de thanmons

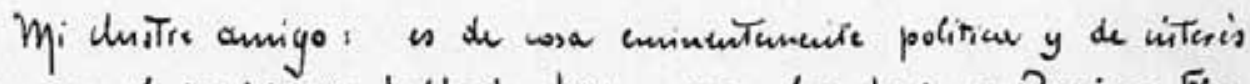

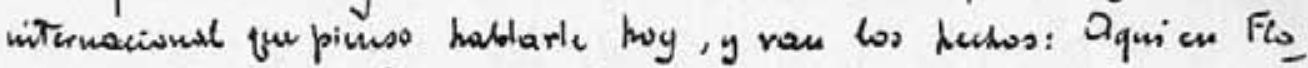

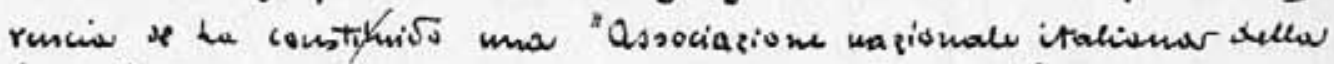

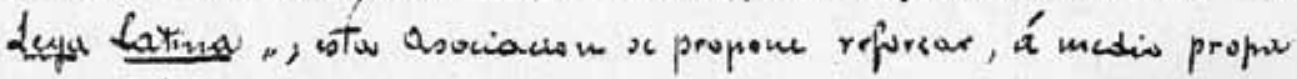

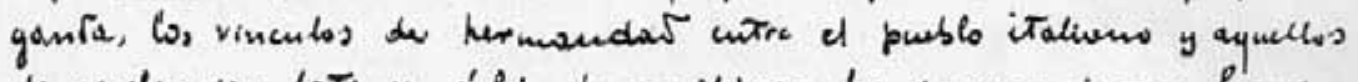
de civilizasion latinu, áfin de equilibrar los ayropanicatos forring dables otue van formands un Europor puebolos estaves y alumanes.

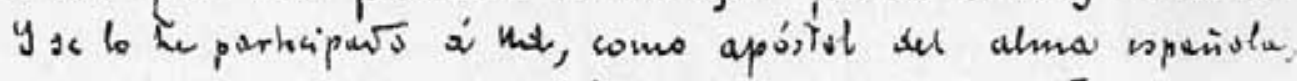

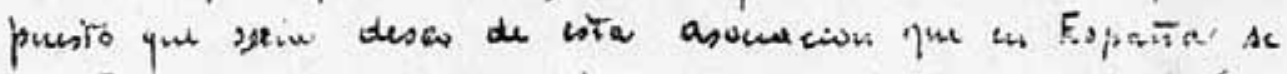

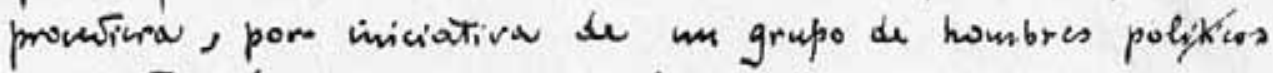
precavisos, á colenticas oryanización.

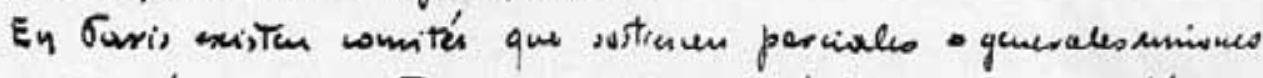

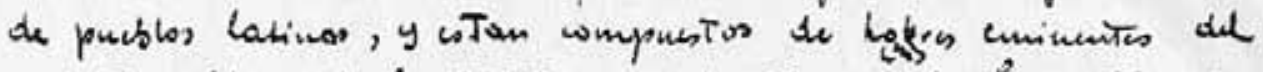

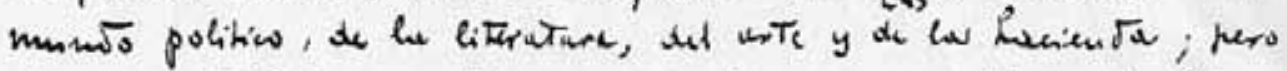

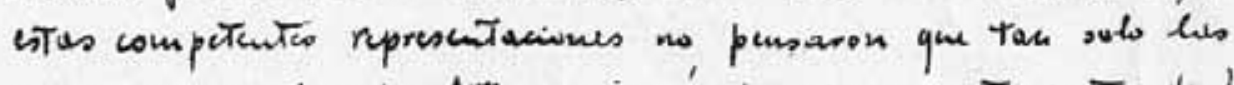

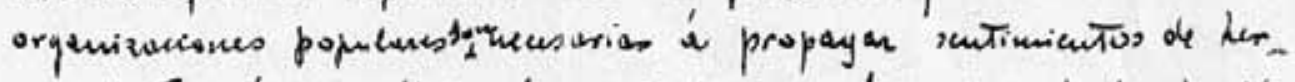

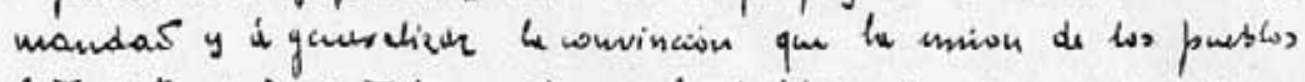

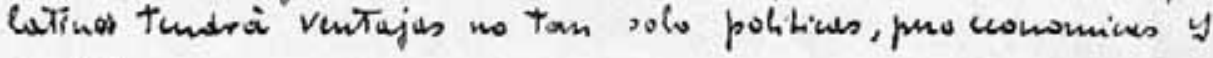
sociales.

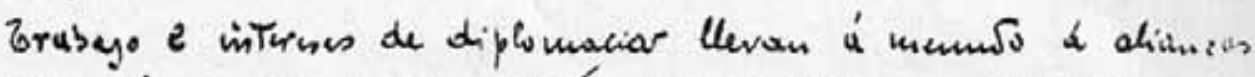

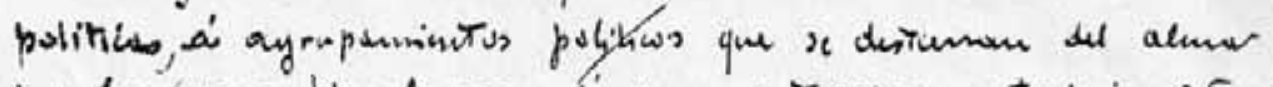
popular o mas bien le son I' vices contrarias: trabajo er

Illustrazioni.- Fac-simile della lettera di Beccari a Unamuno. Lettera inedita, datata 29 maggio, 1913, conservata nell' archivio della casa museo Unamuno di Salamanca.Vedi nota 18. 


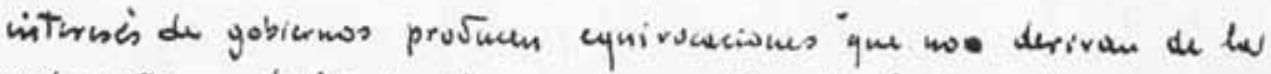
vida litima de los publos, y sirven ton soto à desviertos an now

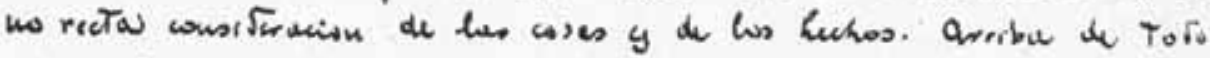

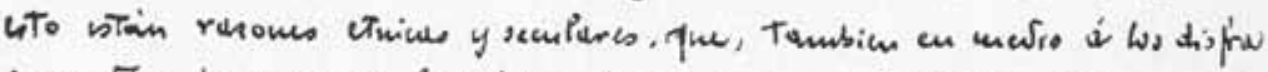

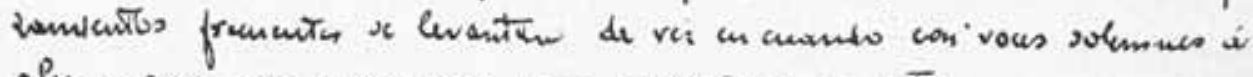
afirmasciones que so nuesurio coyn y Tresucir en acto.

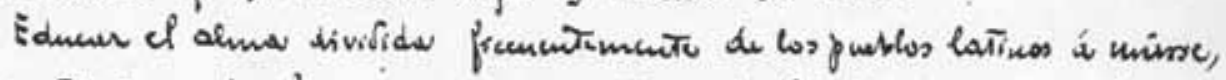

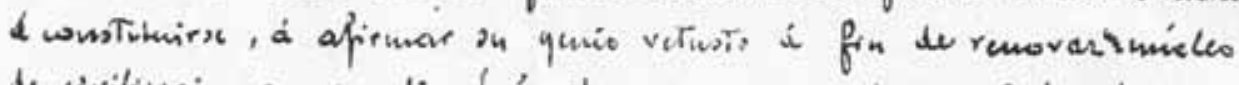

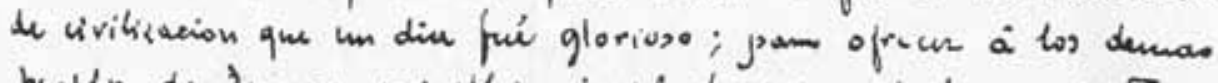

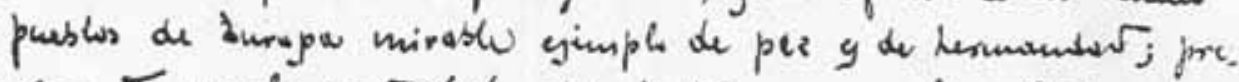

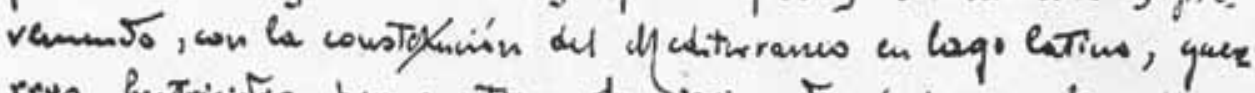
reses pratricisos por uncition de primato, ś por canfines de

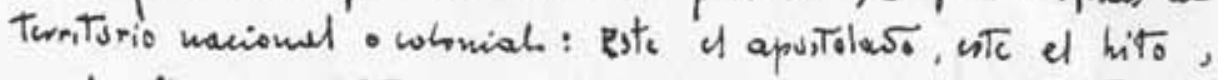

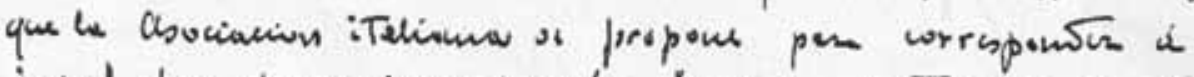

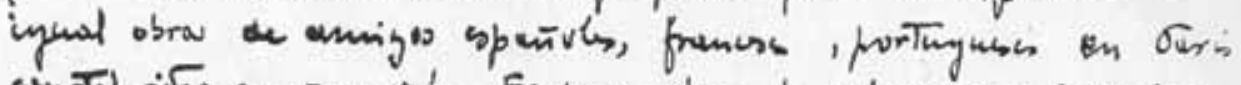

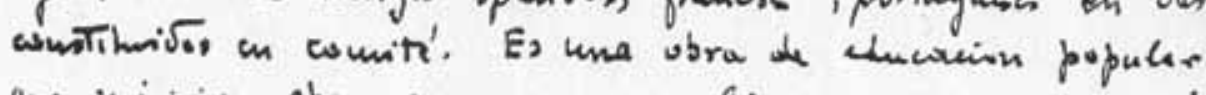
que 2 istisias, obra suyura en no kijuns porrenis contra los mavisbras der las siplonuscios.

I para corresponder , como ya le dije. al derw de boTos

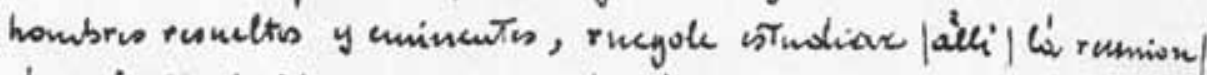
de valientin politicos quicues, subre las normas del staturo ita hion aqui idjunto, procesinem on España a identian organ, zavion. Ai Md. aceptara nTt unamiaso pusienas gustose a ud y í

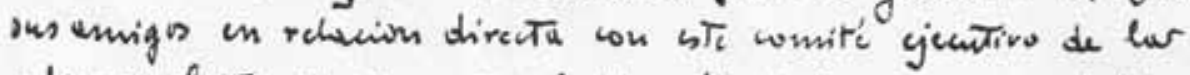

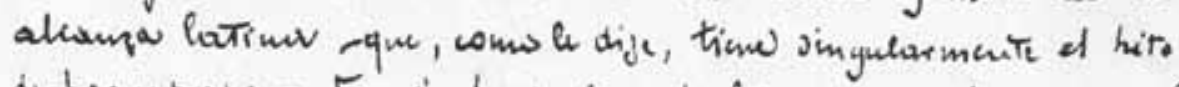
di haew propayantw a he union de has naciones hermancas, á

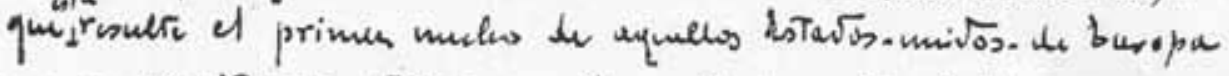

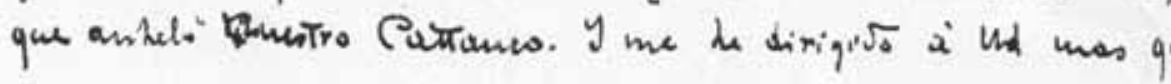




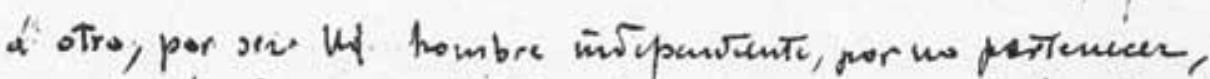

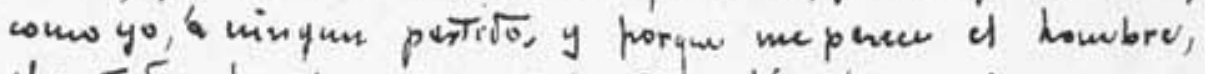

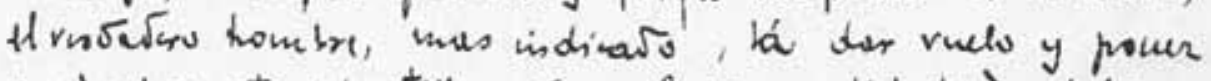

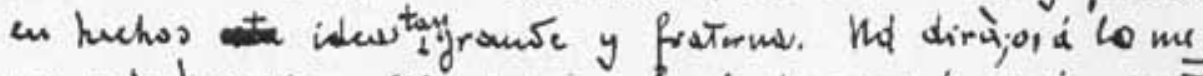

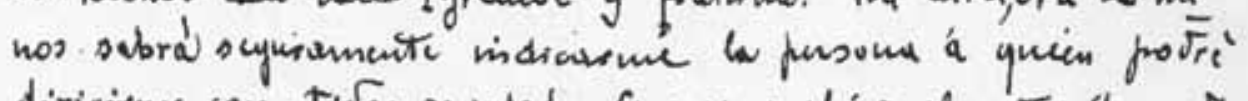
dirigirmucon to $\sqrt{a}$ suiedad. Gracius unil iguslencintc. Clyuarios

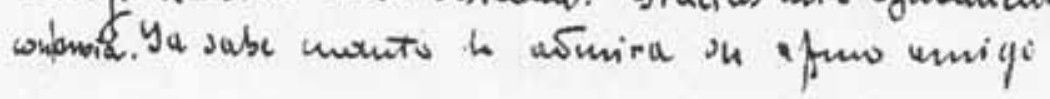
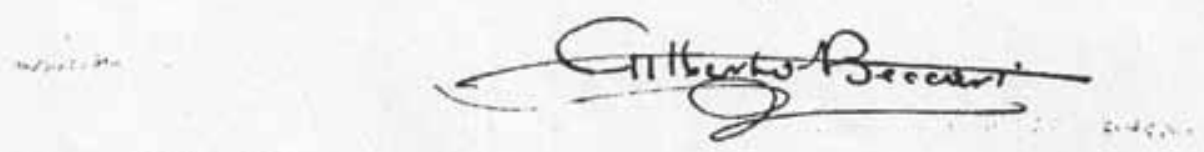

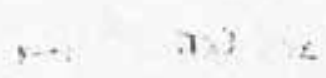

con,

i) 